\title{
Some Recent Results on Models of Dynamical Electroweak Symmetry Breaking
}

\author{
R. Shrock* \\ C. N. Yang Institute for Theoretical Physics \\ State University of New York \\ Stony Brook, New York 11794, USA \\ *email: robert.shrock@sunysb.edu
}

We review some recent results on models of dynamical electroweak symmetry breaking involving extended technicolor.

\section{Introduction}

The origin of electroweak symmetry breaking (EWSB) is an outstanding unsolved question in particle physics. In the Standard Model with gauge group $G_{S M}=\mathrm{SU}(3)_{c} \times \mathrm{SU}(2)_{L} \times \mathrm{U}(1)_{Y}$, this symmetry breaking is produced by postulating a Lorentz scalar Higgs field $\phi=\left(\begin{array}{c}\phi^{+} \\ \phi^{0}\end{array}\right)$ with weak isospin $I=1 / 2$ and weak hypercharge $Y=1$ and assuming that its potential, $V=\mu^{2} \phi^{\dagger} \phi+\lambda\left(\phi^{\dagger} \phi\right)^{2}$, has $\mu^{2}<0$, thereby leading to a nonzero vacuum expection value for $\phi$. However, this mechanism is unsatisfying for several reasons: (i) the EWSB is put in by hand and no explanation is provided as to why $\mu^{2}$ is negative when, a priori, it could be positive; (ii) $\mu^{2}$ and hence $m_{H}^{2}=-2 \mu^{2}=2 \lambda v^{2}$ are unstable to large radiative corrections from much higher energy scales (the gauge hierarchy problem), so that extreme finetuning is needed to keep the Higgs mass of order the electroweak scale; (iii) the SM accomodates, but does not explain, fermion masses and mixing, via Yukawa couplings of the generic form (suppressing the matrix structure) $m_{f} \simeq y_{f} v / \sqrt{2}$; the $y_{f}$ values and generational hierarchy are put in by hand with some $y_{f}$ 's ranging down to $10^{-5}$ with no explanation.

These facts have motivated an alternative approach based on dynamical electroweak symmetry breaking driven by a strongly coupled vectorial gauge interaction, associated with an exact gauge symmetry, called technicolor (TC) [1]. The EWSB is produced by the formation of bilinear condensates of technifermions. To communicate this symmetry breaking to the 
SM fermions (which are technisinglet), one embeds technicolor in a larger, extended technicolor (ETC) theory $[2,3]$. In this talk we will review some of our recent results in this area [4]- [14] obtained in collaboration with T. Appelquist, M. Piai, N. Christensen, and M. Kurachi. (The work with Kurachi is also discussed in his talk [15].)

As further motivation, one may recall that in both of two major previous cases where fundamental scalar fields were used to model spontaneous symmetry breaking, the actual underlying physics did not involve fundamental scalar fields but instead a bilinear fermion condensate. The first of these was superconductivity, where the phenomenological Landau-Ginzburg model made use of a complex scalar field $\phi=\rho e^{i \theta}$ with a free energy functional of the form $V=c_{2}|\phi|^{2}+c_{4}|\phi|^{4}$, where $c_{2} \propto\left(T-T_{c}\right.$ ) as $T \rightarrow T_{c}$ (with $T_{c}$ being the critical temperature). Hence, for $T<T_{c}, c_{2}<0$ and $\langle\phi\rangle \neq 0$. However, the true origin of superconductivity is the dynamical formation of a condensate of Cooper pairs, $\langle e e\rangle$. A second example is spontaneous chiral symmetry breaking in hadronic physics. In the Gell-Mann Lévy $\sigma$ model for this phenomenon, the chiral symmetry breaking is a result of the coefficient of the quadratic term in the potential being arbitrarily chosen to be negative, producing a nonzero vev of the $\sigma$ field, quite analogous to the Higgs mechanism. However, the real origin of spontaneous chiral symmetry breaking in quantum chromodynamics (QCD) is the dynamical formation of a bilinear quark condensate $\langle\bar{q} q\rangle$. Perhaps these previous examples might serve as a guide for thinking about the physics underlying EWSB.

Actually, one already knows of a source of dynamical electroweak symmetry breaking, namely QCD. Consider, for simplicity, QCD with $N_{f}=2$ quarks, $u, d$, taken to be massless. The quark condensate $\langle\bar{q} q\rangle=\left\langle\bar{q}_{L} q_{R}\right\rangle+$ $\left\langle\bar{q}_{R} q_{L}\right\rangle$, transforms as $I_{w}=1 / 2,|Y|=1$. By itself this theory would produce nonzero $W$ and $Z$ masses $m_{W}^{2} \simeq\left(g^{2} / 4\right) f_{\pi}^{2}, m_{Z}^{2} \simeq(1 / 4)\left(g^{2}+g^{\prime 2}\right) f_{\pi}^{2}$. With $f_{\pi}=92 \mathrm{MeV}$, this yields $m_{W} \simeq 30 \mathrm{MeV}, m_{Z} \simeq 34 \mathrm{MeV}$. These $W$ and $Z$ masses satisfy the tree-level relation $\rho=1$, where $\rho=m_{W}^{2} /\left(m_{Z}^{2} \cos ^{2} \theta_{W}\right)$. While the scale here is too small by $\sim 10^{3}$ to explain the observed $W$ and $Z$ masses, it suggests how to construct a model with dynamical EWSB.

We shall consider a technicolor theory with gauge group $\mathrm{SU}\left(N_{T C}\right)$ and a set of technifermions, generically denoted $F$, with zero Lagrangian masses, transforming according to the fundamental representation of the group. The scale of confinement and chiral symmetry breaking in this theory is denoted $\Lambda_{T C}$ and is of order the electroweak scale. One assigns the $\mathrm{SU}(2)_{L}$ representations of the technifermions so that their left and right components form $\mathrm{SU}(2)_{L}$ doublets and singlets, respectively. A minimal choice is the "one- 
doublet" model, with $\left(\begin{array}{c}F_{u}^{\tau} \\ F_{d}^{\tau}\end{array}\right)$ and $F_{u R}^{\tau}, F_{d R}^{\tau}$, where $\tau$ is a TC gauge index and $Y=0(Y= \pm 1)$ for the $\mathrm{SU}(2)_{L}$ doublet (singlets). Since the technicolor theory is asymptotically free, it follows that, as the energy scale decreases, $\alpha_{T C}$ increases, eventually producing condensates $\left\langle\bar{F}_{u} F_{u}\right\rangle$ and $\left\langle\bar{F}_{d} F_{d}\right\rangle$ transforming as $I_{w}=1 / 2,|Y|=1$, breaking EW sym. at $\Lambda_{T C}$. It follows that, to leading order, $m_{W}^{2}=g^{2} f_{T C}^{2} N_{D} / 4$ and $m_{Z}^{2}=\left(g^{2}+g^{\prime 2}\right) f_{T C}^{2} N_{D} / 4$, where $f_{T C} \lesssim \Lambda_{T C}$ is the TC analogue of $f_{\pi} \lesssim \Lambda_{Q C D}$ and $N_{D}$ is the number of $\mathrm{SU}(2)_{L}$ technidoublets $\left(N_{D}=1\right.$ in this case). These masses satisfy the tree-level relation $\rho=1$ [16]. For this minimal example, $f_{T C} \simeq 250 \mathrm{GeV}$. Another class of TC models uses one SM family of technifermions [17],

$$
\begin{array}{ll}
\left(\begin{array}{c}
U^{a \tau} \\
D^{a \tau}
\end{array}\right)_{L}, & U_{R}^{a \tau}, \quad D_{R}^{a \tau} \\
\left(\begin{array}{c}
N^{\tau} \\
E^{\tau}
\end{array}\right)_{L}, & N_{R}^{\tau}, \quad E_{R}^{\tau}
\end{array}
$$

(where $a$ and $\tau$ are color and technicolor indices, respectively) with the usual $Y$ assignments. Again, there is technifermion condensate formation, with approximately equal condensates $\langle\bar{F} F\rangle$ for $F=U^{a}, D^{a}, N, E$, generating dynamical technifermion masses $\Sigma_{T C} \sim \Lambda_{T C}$. In this class of models $N_{D}=$ $N_{c}+1=4$, so $f_{T C} \simeq 125 \mathrm{GeV}$.

Technicolor has the potential to solve/explain various problematic and/or mysterious features of the Standard Model: (i) given the asymptotic freedom of the TC theory, the condensate formation and hence EWSB are automatic and do not require any ad hoc parameter choice like $\mu^{2}<0$ in the SM; (ii) because TC has no fundamental scalar field, there is no hierarchy problem; (iii) because $\langle\bar{F} F\rangle=\left\langle\bar{F}_{L} F_{R}\right\rangle+\left\langle\bar{F}_{R} F_{L}\right\rangle$, technicolor explains why the chiral part of $G_{S M}$ is broken and the residual exact gauge symmetry, $\mathrm{SU}(3)_{c} \times \mathrm{U}(1)_{e m}$, is vectorial. The fact that this latter symmetry is vectorial is, of course, crucial in allowing nonzero mass terms for fermions that are nonsinglets under color or charge.

In order to give masses to quarks and leptons, one must communicate the EWSB in the technicolor sector to these SM fermions, and hence, as mentioned above, one must embed technicolor in the larger ETC theory, with ETC gauge bosons $V_{\tau}^{i}$ transforming (technisinglet) SM fermions into technifermions and vice versa. To satisfy constraints on flavor-changing neutral current (FCNC) processes, the ETC gauge bosons must have large masses. These masses can arise from self-breaking (tumbling) of the ETC chiral gauge symmetry. The ETC theory is arranged to be asymptotically free, so 
as the energy decreases from a high scale, the ETC coupling $\alpha_{E T C}$ grows and eventually becomes large enough to form condensates which sequentially break the ETC symmetry group down at scales $\Lambda_{j}, j=1,2,3$ to a residual exact TC subgroup, where

$$
\Lambda_{1} \simeq 10^{3} \mathrm{TeV}, \quad \Lambda_{2} \simeq 50-100 \mathrm{TeV}, \quad \Lambda_{3} \simeq \text { few TeV },
$$

We will mainly focus on $\mathrm{SU}\left(N_{E T C}\right)$ ETC models with one-family TC. These gauge the Standard Model fermion generation index and combine it with the TC index $\tau$, so

$$
N_{E T C}=N_{\text {gen. }}+N_{T C}=3+N_{T C} .
$$

To be viable, modern TC models are designed to have a coupling $g_{T C}$ that gets large, but runs slowly ("walks") over an extended interval of energy (WTC) [18]- [24]. For sufficiently many technifermions, the TC beta function has a second zero (approximate infrared fixed point of the renormalization group) at a certain $\alpha_{T C}=\alpha_{I R} \neq 0$. As the number of technifermions, $N_{f}$, increases, $\alpha_{I R}$ decreases. In WTC, one arranges so that $\alpha_{I R}$ is slightly greater than the critical value, $\alpha_{c r}$, for $\langle\bar{F} F\rangle$ formation. As $N_{f} \nearrow N_{f, c r}, \alpha_{I R} \searrow \alpha_{c r}$. Combining the calculation of $\alpha_{I R}$ from the two-loop beta function and an estimate of $\alpha_{c r}$ from the Schwinger-Dyson equation, one finds $N_{f, c r}=2 N_{T C}\left(50 N_{T C}^{2}-33\right) /\left[5\left(5 N_{T C}^{2}-3\right)\right]$ [22]. Hence, for $N_{T C}=2$, one has $N_{f, c r} \simeq 8$. Thus, if $N_{T C}=2$, a one-family TC theory, with its $N_{f}=N_{w}\left(N_{c}+1\right)=8$ technifermions plausibly exhibits walking behavior.

In a walking TC theory, as energy scale decreases, $\alpha_{T C}$ grows, but its rate of increase, $|\beta|$, decreases toward zero as $\alpha_{T C} \nearrow \alpha_{I R}$, where $\beta=0$. Eventually, $\alpha_{T C}$ exceeds $\alpha_{c r},\langle\bar{F} F\rangle$ forms, and the technifermions gain dynamical masses $\Sigma_{T C} \simeq \Lambda_{T C}$. WTC has several advantages: (i) SM fermion masses are enhanced by the factor $\eta=\exp \left[\int_{\Lambda_{T C}}^{\Lambda_{w}} \mu^{-1} d \mu \gamma\left(\alpha_{T C}(\mu)\right)\right]$; (ii) hence, one can increase ETC scales $\Lambda_{i}$ for a fixed $m_{f_{i}}$, reducing FCNC effects; (iii) $\eta$ also enhances masses of pseudo-Nambu Goldstone bosons (PNGB's); and (iv) the walking can reduce the value of the $S$ parameter [25], given by

$$
\frac{\alpha_{e m} S}{\sin ^{2}\left(2 \theta_{W}\right)}=\frac{\Pi_{Z Z}\left(m_{Z}^{2}\right)-\Pi_{Z Z}(0)}{m_{Z}^{2}} .
$$

The value $N_{T C}=2$ is also motivated by the fact that it makes possible a mechanism to explain light neutrinos in (E)TC $[4,5]$. Substituting this value $N_{T C}=2$ into eq. (4) yields $N_{E T C}=5$. 


\section{ETC Models}

TC/ETC models are very ambitious, since a successful model would explain not only EWSB but also the spectrum of Standard Model fermion masses. Thus it is perhaps not surprising that no fully realistic model of this type has been constructed yet. These models are subject to several strong constraints from neutral flavor-changing current processes and precision electroweak data. We have studied the properties of several types of ETC models in our work. One of these has a gauge group $G=\mathrm{SU}(5)_{E T C} \times \mathrm{SU}(2)_{H C} \times G_{S M}$. In addition to ETC, this has another gauge interaction, hypercolor (HC), which helps to produce the desired ETC symmetry-breaking pattern. The SM fermions and corresponding technifermions transform according to the representations

$$
\begin{gathered}
Q_{L}:(5,1,3,2)_{1 / 3, L}, \quad u_{R}:(5,1,3,1)_{4 / 3, R}, \quad d_{R}:(5,1,3,1)_{-2 / 3, R}, \\
L_{L}:(5,1,1,2)_{-1, L}, \quad e_{R}:(5,1,1,1)_{-2, R},
\end{gathered}
$$

where the subscripts denote $Y$. For example, writing out the components of $e_{R}$, one has $\left(e^{1}, e^{2}, e^{3}, e^{4}, e^{5}\right)_{R} \equiv\left(e, \mu, \tau, E^{4}, E^{5}\right)_{R}$, where the last two entries are the charged technileptons. In addition, the model includes the SM-singlet ETC-nonsinglet fermions

$\psi_{i j, R}:(\overline{10}, 1,1,1)_{0, R}, \quad \zeta_{R}^{i j, \alpha}:(10,2,1,1)_{0, R}, \quad \omega_{p, R}^{\alpha}: 2(1,2,1,1)_{0, R}$

where here $1 \leq i, j \leq 5$ are $\mathrm{SU}(5)_{E T C}$ indices, $\alpha=1,2$ is an $\mathrm{SU}(2)_{H C}$ index, and $p=1,2$ is a copy number. In this model the SM fermions and corresponding technifermions have vectorial couplings to ETC gauge bosons, but the SM-singlet sector makes the full ETC theory a chiral gauge theory. Hence, there are no fermion mass terms in the lagrangian. This theory has no gauge or global anomalies.

Since the ETC theory is asymptotically free, its gauge coupling grows as the energy scale decreases. The ETC breaking occurs because of the formation of ETC-noninvariant bilinear condensates of ETC-nonsinglet, SM-singlet fermions. To analyze the stages of symmetry breaking, we identify plausible preferred condensation channels using a generalized most-attractive-channel (MAC) approach. We envision that as the energy decreases from high values down to $E \sim \Lambda_{1} \sim 10^{3} \mathrm{TeV}$, the coupling $\alpha_{E T C}$ becomes sufficiently large to produce condensation in the attractive channel $(\overline{10}, 1,1,1)_{0, R} \times(\overline{10}, 1,1,1)_{0, R} \rightarrow(5,1,1,1)_{0}$, breaking $\mathrm{SU}(5)_{E T C} \rightarrow \mathrm{SU}(4)_{E T C}$. With respect to the unbroken $\mathrm{SU}(4)_{E T C}$, we have 
$(\overline{10}, 1,1,1)_{0, R}=(\overline{4}, 1,1,1)_{0, R}+(\overline{6}, 1,1,1)_{0, R}$; we denote the $(\overline{4}, 1,1,1)_{0, R}$ as $\alpha_{1 i, R} \equiv \psi_{1 i, R}$ for $2 \leq i \leq 5$ and the $(\overline{6}, 1,1,1)_{0, R}$ as $\xi_{i j, R} \equiv \psi_{i j, R}$ for $2 \leq i, j \leq 5$. The associated condensate is then

$$
\left\langle\epsilon^{1 i j k \ell} \xi_{i j, R}^{T} C \xi_{k \ell, R}\right\rangle=8\left\langle\xi_{23, R}^{T} C \xi_{45, R}-\xi_{24, R}^{T} C \xi_{35, R}+\xi_{25, R}^{T} C \xi_{34, R}\right\rangle .
$$

The six fields $\xi_{i j, R}, 2 \leq i, j \leq 5$, involved in this condensate gain dynamical masses $\simeq \Lambda_{1}$.

At energy scales below $\Lambda_{1}$, depending on relative strengths of gauge couplings, different symmetry-breaking sequences can occur. Again, these arise via the dynamical formation of condensates involving SM-singlet ETC-nonsinglet fermions. For example, one sequence, S1, leads to the breaking $\mathrm{SU}(4)_{E T C} \rightarrow \mathrm{SU}(3)_{E T C}$ at $\Lambda_{2} \simeq 50-100 \mathrm{TeV}$ and finally $\mathrm{SU}(3)_{E T C} \rightarrow \mathrm{SU}(2)_{T C}$ at $\Lambda_{3} \simeq$ few TeV, with $\mathrm{SU}(2)_{H C}$ unbroken. Another sequence, $\mathrm{S} 2$, involves a breaking $\mathrm{SU}(4)_{E T C} \rightarrow \mathrm{SU}(2)_{E T C}$ at $\Lambda_{23} \simeq 50 \mathrm{TeV}$ and $\mathrm{SU}(2)_{H C} \rightarrow \mathrm{U}(1)_{H C}$ at a scale $\Lambda_{B H C} \lesssim \Lambda_{1}$. In all cases, $\mathrm{SU}(2)_{T C}$ is an exact gauge symmetry. At the lowest scale, $\Lambda_{T C}$, the technifermion condensates form, breaking electroweak symmetry and giving masses to the $W$ and $Z$.

Certain fermion condensates contribute to nondiagonal ETC gauge boson propagator corrections and hence mixing. For example, in sequence $S_{1}$, the mixing $V_{1}^{\tau} \rightarrow V_{3}^{\tau}, \tau=4,5$ is induced by the graph in Fig. 1, with the

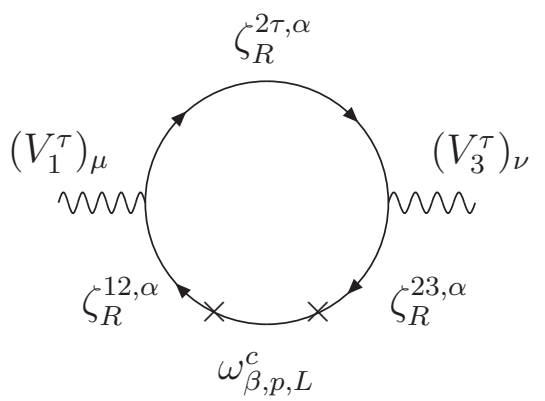

Fig. 1. Graph for $V_{1}^{\tau} \rightarrow V_{3}^{\tau}$ with $\tau=4,5$, for sequence S1.

result

$$
{ }_{3}^{\tau} \Pi_{1}^{\tau}(0) \simeq \frac{\alpha_{E T C}}{4 \pi} \int\left(k^{2} d k^{2}\right) \frac{k^{4} \Sigma_{3}(k)^{2}}{\left[k^{2}+\Sigma_{3}(k)^{2}\right]^{4}},
$$

where $\Sigma_{3} \simeq \Lambda_{3}$. This yields ${ }_{3}^{\tau} \Pi_{1}^{\tau}(0) \simeq$ const. $\times \Lambda_{3}^{2}$. In sequence $S_{2}$, the mixing $V_{2}^{\tau} \rightarrow V_{3}^{\tau}, \tau=4,5$ is induced by the graph in Fig. 2, giving ${ }_{3}^{\tau} \Pi_{2}^{\tau}(0) \simeq$ 


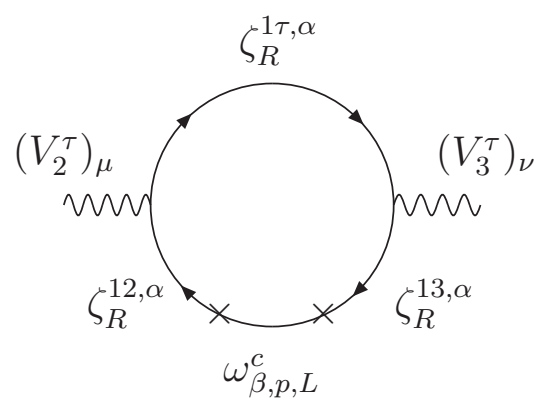

Fig. 2. Graph for $V_{2}^{\tau} \rightarrow V_{3}^{\tau}$ with $\tau=4,5$, for sequence S2.

const. $\times \Lambda_{23}^{2}$. We find that the feature that nondiagonal ETC gauge boson propagator corrections ${ }_{i}^{\tau} \Pi_{j}^{\tau}(0)$ are proportional to the square of the lowest ETC scale (or smaller) is generic in this type of ETC model, reflecting a type of approximate generational symmetry. Other ETC gauge boson mixings are similarly suppressed.

\section{Fermion Masses and Mixing}

Figure 3 shows a one-loop graph contributing to the mass matrix element $M_{i j}^{(f)}$ for a SM fermion $f$ (up- or down-type quark or charged lepton) appearing in the operator $\bar{f}_{i, L} M_{i j}^{(f)} f_{j, R}+$ h.c.. In this figure we distinguish the first three ETC indices, which refer to SM fermion generations, and the indices 4,5 which are TC, by denoting the latter as $\tau=4,5$. An estimate

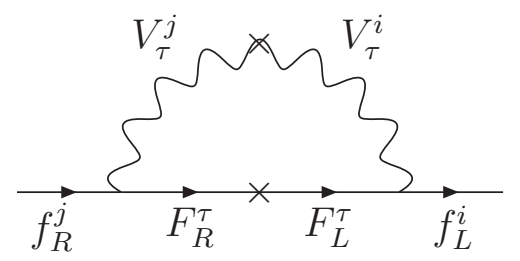

Fig. 3. Graph contributing to the fermion mass matrix element $M_{i j}^{(f)}$. 
for diagonal entries is (with no sum on $i$ )

$$
M_{i i}^{(f)} \simeq \frac{\kappa\left(N_{T C} / 2\right) \eta \Lambda_{T C}^{3}}{\Lambda_{i}^{2}},
$$

where $\kappa \simeq O(10)$ is a numerical factor from the integral and in WTC, $\eta \simeq \Lambda_{3} / \Lambda_{T C}$. This is only a rough estimate, since ETC coupling is strong, so higher-order diagrams are also important. The sequential breaking of the ETC symmetry at the highest scale $\Lambda_{1}$, the intermediate scale $\Lambda_{2}$, and the lowest scale $\Lambda_{3}$, thus produces the generational hierarchy in the fermion masses.

Insertions of the nondiagonal ETC propagator corrections (indicated by the cross on the gauge boson line in Fig. 3) give rise to off-diagonal elements of the $M^{(f)}$. These have the form

$$
M_{i j}^{(f)} \simeq \frac{\kappa \eta \Lambda_{T C}^{3}{ }_{\tau}^{j} \Pi_{\tau}^{i}}{\Lambda_{i}^{2} \Lambda_{j}^{2}} .
$$

Although the resultant mixing is not fully realistic, this model shows how not just diagonal but also off-diagonal elements of SM fermion mass matrices $M^{(f)}$ and hence CKM mixing, could arise dynamically [6]. Further corrections to $M^{(f)}$ arise from SM gauge interactions, in particular, $\mathrm{SU}(3)_{c}$ and $\mathrm{U}(1)_{Y}$, and from direct diagonal ETC gauge boson exchanges, in particular, $V_{d 3}$ (having mass $\sim \Lambda_{3}$ and corresponding to the $\mathrm{SU}(5)_{E T C}$ generator $\left.T_{d 3}=(2 \sqrt{3})^{-1} \operatorname{diag}(0,0,-2,1,1)\right)$.

\section{Mechanism for Light Neutrinos}

An old puzzle in TC/ETC theories was how to explain light neutrino masses. A solution to this was given in [4] and analyzed further in $[5,6]$. The $\alpha_{1 j, R}$ with $j=2,3$ are right-handed electroweak-singlet neutrinos and get induced Dirac neutrino mass terms connecting with $\left(n^{1}, n^{2}, n^{3}\right)_{L}=$ $\left(\nu_{e}, \nu_{\mu}, \nu_{\tau}\right)_{L}$. These Dirac masses $\bar{n}_{i, L} M_{D} \alpha_{1 j, R}+$ h.c. cannot be generated by the usual one-loop ETC graphs that produce diagonal quark and charged lepton masses and are thus suppressed. The $\alpha_{1 j, R}$ also have induced Majorana mass terms $\alpha_{1 i, R}^{T} C r_{i j} \alpha_{1 j, R}+$ h.c. For example, with sequence S2, denoting the relevant Dirac mass terms as $b_{i j}$, one finds $b_{i j} \simeq \kappa \Lambda_{T C}^{4} / \Lambda_{23}^{3} \simeq$ $O(0.1) \mathrm{MeV}$ for $2 \leq i, j \leq 3$; further, $r_{23} \simeq \kappa \Lambda_{B H C}^{3} \Lambda_{23}^{3} / \Lambda_{1}^{5}$. Numerically, $\left|r_{23}\right| \simeq O\left(10^{2}\right) \mathrm{GeV}$. The resultant electroweak-nonsinglet neutrinos are, to very good approximation, linear combinations of three mass eigenstates, with $\nu_{3}$ mass

$$
m\left(\nu_{3}\right) \simeq \frac{\left(b_{23}+b_{22}\right)^{2}}{r_{23}} \simeq \frac{\kappa \Lambda_{T C}^{8} \Lambda_{1}^{5}}{\Lambda_{23}^{9} \Lambda_{B H C}^{3}}
$$


With the above-mentioned numerical values and $\Lambda_{B H C} \lesssim \Lambda_{1}$, we find $m\left(\nu_{3}\right) \simeq 0.05 \mathrm{eV}$, consistent with experimental results on neutrino oscillations. Similarly,

$$
\frac{m\left(\nu_{2}\right)}{m\left(\nu_{3}\right)}=\left(\frac{b_{23}-b_{22}}{b_{23}+b_{22}}\right)^{2}
$$

which is again consistent with experimental data. Since $\left|r_{23}\right|>>\left|b_{i j}\right|$, this is a seesaw, but quite different from the SUSY GUT seesaw; the Majorana masses $r_{i j}$ that underly the seesaw are not GUT-scale and are actually much smaller than the ETC scales $\Lambda_{i}$.

\section{Constraints from Neutral Flavor-Changing Current Processes}

An early concern was that ETC interactions would lead to excessively large flavor-changing neutral current processes. A particularly severe constraint arises from $K^{0}-\bar{K}^{0}$ mixing. Early treatments wrote the effective Lagrangian for this as

$$
\mathcal{L}_{e f f} \simeq \frac{1}{\Lambda_{E T C}^{2}}\left[\bar{s} \gamma_{\mu} d\right]^{2},
$$

where $\Lambda_{E T C}$ was a generic ETC scale. To suppress FCNC effects adequately, it was thought that $\Lambda_{E T C}$ had to be so high that there would be excessive suppression of SM fermion masses. However, we have shown, using our reasonably UV-complete theories, that in the present type of ETC theory this old view was too pessimistic; ETC contributions to FCNC processes are smaller than had been inferred with the above naive $\mathcal{L}_{\text {eff }}$, because of residual approximate generational symmetries [6]- [8].

The coupling of the ETC gauge bosons to the SM fermion mass eigenstates is given by

$$
\mathcal{L}_{i n t}=g_{E T C} \sum_{f, j, k} \bar{f}_{j} \gamma_{\lambda}\left(\mathcal{V}^{\lambda}\right)_{k}^{j} f^{k} \equiv g_{E T C} \sum_{f, j, k} \bar{f}_{m, j} \gamma_{\lambda}\left(A^{\lambda}\right)_{k}^{j} f_{m}^{k},
$$

where $\mathcal{V}$ is a matrix containing the ETC gauge fields, $A^{\lambda} \equiv U^{(f)} \mathcal{V}^{\lambda} U^{(f)}-1$, and the $U^{(f)}$ are the unitary transformations that diagonalize $M^{(f)}$ via $U^{(f)} M^{(f)} U^{(f)-1}=M_{\text {diag. }}^{(f)}$. We parametrize each $U^{(f)}$ with a PDG-type parametrization in terms of $\theta_{12}^{(f)}, \theta_{13}^{(f)}, \theta_{23}^{(f)}$, and $\delta^{(f)}$. The approximate generational symmetry in the ETC sector can naturally yield relatively small CKM mixing with $\theta_{j k}^{(f)}$ depending on ratios of smaller to larger ETC scales.

We have analyzed ETC contributions to a number of processes, including the neutral meson mixings $K^{0}-\bar{K}^{0}, D^{0}-\bar{D}^{0}, B_{d}^{0}-\bar{B}_{d}, B_{s}^{0}-\bar{B}_{s}$, and 
decays such as $\mu^{+} \rightarrow e^{+} e^{+} e^{-}$, involving four-fermion operators [6,8]. As an example, consider $K^{0}-\bar{K}^{0}$ mixing and the resultant $K_{L}-K_{S}$ mass difference $\Delta m_{K_{L} K_{S}}$. The SM contribution is consistent with the experimental value, $\Delta m_{K_{L} K_{S}} / m_{K}=0.70 \times 10^{-14}$. A key to the suppression is the fact that, in terms of ETC eigenstates, an $s \bar{d}$ in a $\bar{K}^{0}$ produces a $V_{1}^{2}$ ETC gauge boson, but this cannot directly yield a $d \bar{s}$ in the final-state $K^{0}$; the latter is produced by a $V_{2}^{1}$. Hence, this requires either the ETC gauge boson mixing $V_{1}^{2} \rightarrow V_{2}^{1}$ or the mixing of ETC quark eigenstates to produce mass eigenstates. The contribution from $V_{1}^{2} \rightarrow V_{2}^{1}$ yields a coefficient

$$
c \sim \frac{1}{\Lambda_{1}^{2}}{ }_{2}^{1} \Pi_{1}^{2} \frac{1}{\Lambda_{1}^{2}} \sim \frac{\Lambda_{3}^{2}}{\Lambda_{1}^{2}} \frac{1}{\Lambda_{1}^{2}} \ll \frac{1}{\Lambda_{1}^{2}} .
$$

With above values, $\Lambda_{1} \sim 10^{3} \mathrm{TeV}, \Lambda_{3} \sim 3 \mathrm{TeV}$, the suppression factor is $\left(\Lambda_{3} / \Lambda_{1}\right)^{2} \simeq 10^{-5}$. So, rather than the naive result $\Delta m_{K_{L} K_{S}} / m_{K} \sim \Lambda_{Q C D}^{2} / \Lambda_{1}^{2}$, this yields the much smaller result $\Delta m_{K_{L} K_{S}} / m_{K} \sim \Lambda_{3}^{2} \Lambda_{Q C D}^{2} / \Lambda_{1}^{4} \sim 10^{-18}$. Hence, the dominant ETC contributions arise from the mixing of ETC eigenstates of quarks to form mass eigenstates. First, $s \bar{d}$ can couple to $V_{d 2}$ (having mass $\simeq \Lambda_{2}$ and corresponding to the $\mathrm{SU}(5)_{E T C}$ generator $\left.T_{d 2}=(2 \sqrt{6})^{-1} \operatorname{diag}(0,-3,1,1,1)\right)$, with coefficient $\theta_{12}^{(d)}$. The resultant diagram with exchange of this gauge boson $V_{d 2}$ contributes $\sim\left(\theta_{12}^{(d)}\right)^{2} / \Lambda_{2}^{2}$ to the amplitude. Requiring this to be small relative to SM contribution yields the constraint $\left|\theta_{12}^{(d)}\right| \lesssim 10^{-2}$. Second, $s \bar{d}$ can couple to $V_{d 3}$, with coefficient $\theta_{13}^{(d)} \theta_{23}^{(d)}$. The resultant diagram contributes $\sim\left(\theta_{13}^{(d)} \theta_{23}^{(d)}\right)^{2} / \Lambda_{3}^{2}$ to the amplitude. This yields the constraint $\left|\theta_{13}^{(d)} \theta_{23}^{(d)}\right| \lesssim 0.4 \times 10^{-3}$. A comprehensive analysis of constraints from processes involving dimension-six four-fermion operators was given in $[6,8]$. A related analysis is [26].

We have also studied ETC contributions to (dimension-5) diagonal and transition lepton and quark dipole moments, and constraints from limits on $\mu^{+} \rightarrow e^{+} \gamma, \tau^{+} \rightarrow \ell^{+} \gamma$, the measured muon $(g-2)$ and $b \rightarrow s \gamma$ decay, and limits on lepton, neutron, atomic electric dipole moments [7]. Again, we found that this type of ETC model can be consistent with existing data.

\section{Precision Electroweak Constraints}

One may ask what the momentum dependence of a SM fermion mass is in this type of theory. This question was answered in [9]; the running mass $m_{f_{j}}(p)$ exhibits the power-law decay

$$
m_{f_{j}}(p) \propto \frac{\Lambda_{j}^{2}}{p^{2}}
$$


for Euclidean momenta $p \gg \Lambda_{j}$, where $f_{j}$ is a fermion of generation $j$. (Here we neglect logarithmic factors, which are subdominant relative to this power-law falloff.) Thus, $m_{t}(p)$ and $m_{b}(p)$ decay like $\Lambda_{3}^{2} / p^{2}$ for $p \gg \Lambda_{3}$, while $m_{u}(p)$ and $m_{d}(p)$ are hard up to the much higher scale $\Lambda_{1}$. We have investigated whether precision electroweak data are consistent with these power-law decays of SM fermion masses and have found that they are. The largest effects occur for $m_{t}$. Consider, e.g., the $t$ quark contribution to $\rho$. The conventional (hard, one-loop) result for this is $(\Delta \rho)_{t, h a r d} \simeq 3 G_{f} m_{t}^{2} /\left(8 \pi^{2} \sqrt{2}\right)$. The power-law decay of $m_{t}$ above $\Lambda_{3}$ changes this to $(\Delta \rho)_{t}=(\Delta \rho)_{t, h a r d}\left[1-a_{\rho}\left(m_{t}^{2} / \Lambda_{3}^{2}\right)\right]$, where $a_{\rho}$ is positive, $\sim O(1)$. The softness of $m_{t}$ thus slightly reduces the violation of custodial symmetry. We find a similarly small change in the $(t, b)$ contribution to $S$ relative to the conventional hard-mass result.

As noted, the $S$ parameter places a stringent constraint on TC/ETC theories. A naive perturbative calculation gives, for the TC contribution to $S$, the result $S_{\text {pert. }} \simeq N_{T C} N_{D} /(6 \pi)$, where $N_{D}$ denotes the number of technifermion EW doublets (given that the dynamical masses of the technifermions in each EW doublet are nearly degenerate, as should be true to satisfy the $\rho$-parameter constraint). However, this calculation assumes that the technifermions are weakly interacting, whereas actually, they are strongly interacting, at the relevant scale, $\sim m_{Z}$; hence, this perturbative formula cannot be expected to be reliable. This is important, since otherwise, even with the minimal value, $N_{T C}=2$, it would yield an excessively large value of $S_{\text {pert }}=4 /(3 \pi) \simeq 0.4$ for a one-family TC model (and the marginally acceptable value of $S_{\text {pert. }}=1 /(3 \pi) \simeq 0.1$ for a TC model with one EW technifermion doublet. Experimentally, $S \lesssim 0.1$. Several studies have found that walking can reduce $S$ [27]- [29], [13]. In particular, Refs. [28] and [13] have used solutions of Schwinger-Dyson and Bethe-Salpeter equations to evaluate $S$ [15].

One way to reduce $S$ would be to reconsider TC models with only one technifermion electroweak doublet. But with this technifermion content alone, these models would not have the walking behavior that is needed not just to reduce $S$ but also to generate adequate fermion masses. One can maintain the necessary walking behavior in a TC model with one EW doublet of technifermions by adding a requisite set of SM-singlet technifermions [11]. The $\mathrm{SU}(2)_{T C}$ theory should have $N_{f} \simeq 8$ Dirac technifermions for walking. The electroweak-nonsinglet technifermions comprise two, so we need six more. One adds six SM-singlet, Dirac fermions (i.e., 12 chiral components) that transform as 2's under $\mathrm{SU}(2)_{T C}$. These do not 
contribute to $S$ or $T$. Note that in this theory, $\left[G_{E T C}, G_{S M}\right] \neq 0$, so the ETC gauge bosons carry color and charge. Embedding TC in ETC is thus more complicated than for one-family TC models. Another approach is to use technifermions in higher-dimensional representations [30,31], [11]. In general, the $S$ constraint remains a concern for TC/ETC theories.

\section{Splitting of $t$ and $b$ Masses}

Another challenge for TC/ETC models is to account for the splitting of the $t$ and $b$ masses without excessive contributions to $\rho-1$ (violation of custodial SU(2) symmetry). One cannot do this by having $\Sigma_{T C, U}$ significantly larger than $\Sigma_{T C, D}$ since this would violate the custodial symmetry too much. One could consider trying to achieve this splitting using a class of ETC models in which left and right components of up-type quarks and techniquarks transform the same way under $\mathrm{SU}(5)_{E T C}$, but the left and right components of down-type quarks and techniquarks transform according to relatively conjugate representations. However, we showed that such models have serious problems with excessively large FCNC's [8]. For example, consider a model in which $Q_{L}$ and $u_{R}$ are 5 's of SU(5) ETC and $d_{R}$ is a $\overline{5}$. So, e.g., the $\bar{K}^{0}-K^{0}$ transition can proceed directly via $s_{L} \bar{d}_{L} \rightarrow V_{1}^{2} \rightarrow d_{R} \bar{s}_{R}$ without ETC gauge boson mixing. This gives too large a value for $\Delta m_{K_{L} K_{S}}$.

A different idea would be to use two ETC gauge groups, say SU $(5)_{E T C} \times$ $\mathrm{SU}(5)_{E T C}^{\prime}$ such that the left- and right-handed components of charge $Q=2 / 3$ quarks transform under the same ETC group, while left- and righthanded components of charge $-1 / 3$ quarks and charged leptons transform under different ETC groups $[12,32]$. These models thereby suppress the masses $m_{b}$ and $m_{\tau}$ relative to $m_{t}$, etc. because generating $m_{b}$ requires mixing between the two ETC groups, which is suppressed, while $m_{t}$ does not. However, they tend to produce too much suppression of the masses of firstand second-generation down-type quarks and charged leptons [12]. Thus, a satisfactory explanation of $t-b$ splitting appears to remain a challenge for ETC models.

\section{Dynamical Breaking of Higher Gauge Symmetries}

To what extent can one embed a TC/ETC in a theory having higher gauge symmetry, using dynamical symmetry breaking to break this higher symmetry? Such higher unification would be desirable in order to explain features not explained by the standard model, including charge quantization, prediction of relative sizes of gauge couplings, and unification of quarks and 
leptons. In [5] we constructed asymptotically free gauge theories exhibiting dynamical breaking of the left-right, strong-electroweak gauge group

$$
G_{L R}=\mathrm{SU}(3)_{c} \times \mathrm{SU}(2)_{L} \times \mathrm{SU}(2)_{R} \times \mathrm{U}(1)_{B-L}
$$

(where $B$ and $L$ denote baryon and lepton number) and its extension to

$$
G_{422}=\mathrm{SU}(4)_{P S} \times \mathrm{SU}(2)_{L} \times \mathrm{SU}(2)_{R},
$$

where $\mathrm{SU}(4)_{P S} \supset \mathrm{SU}(3)_{c} \times \mathrm{U}(1)_{B-L}$ is the Pati-Salam group. These models technicolor for electroweak breaking, and extended technicolor for the breaking of $G_{L R}$ and $G_{422}$ and the generation of fermion masses, including a seesaw mechanism for neutrino masses. These models explain why $G_{L R}$ and $G_{422}$ break to $\mathrm{SU}(3)_{c} \times \mathrm{SU}(2)_{L} \times \mathrm{U}(1)_{Y}$, and why this takes place at a scale $\left(\sim 10^{3} \mathrm{TeV}\right)$ which is large compared to the electroweak scale. In particular, the model with the gauge group $G_{422}$ achieves charge quantization in the context of dynamical breaking of all symmetries.

We have also investigated various more ambitious unification schemes involving TC/ETC [10]. In particular, we have studied the possibility of unifying a one-family technicolor group with the SM gauge group in a simple group, but find that it appears difficult to obtain the requisite symmetry breaking dynamically.

\section{Other Phenomenology}

There are several other relevant topics for discussion. One has to do with the spectrum of these theories. As a confining gauge theory, technicolor produces a spectrum of TC-singlet techni-hadrons composed of technifermions and technigluons. This spectrum exhibits differences in a walking theory, as compared with a QCD-like theory. For example, while $m_{a_{1}} / m_{\rho}=1.6$ in QCD, the analogous ratio $m_{\left(a_{1}\right)_{T C}} / m_{\rho_{T C}}$ is close to unity in the walking limit [33]. This is natural, since a theory with walking behavior has $N_{f}$ near to, although slightly less than, the critical value $N_{f, c r}$ at which the theory would go over from the confined phase with spontaneous chiral symmetry breaking to a chirally symmetric phase. It is of interest to investigate how these and other hadron masses change as one decreases $N_{f}$ (hence increases $\alpha_{I R}$ ) to move from the walking regime near $N_{f, c r}$ to-

ward the QCD-like regime at smaller $N_{f}$; this was done in [14]. Searches for the production and decays of these technihadrons at the CERN Large Hadron Collider will be of great importance in testing technicolor theories. For example, by analogy with $\rho \rightarrow \pi \pi$ in QCD, since the technipions are absorbed to become the longitudinal components of the $W$ and $Z$, one 
would have $\rho_{T C}^{0} \rightarrow W_{L}^{+} W_{L}^{-}, \rho_{T C}^{+} \rightarrow W_{L}^{+} Z_{L}^{0}$, etc. A related issue concerns pseudo-Nambu Goldstone bosons. While walking raises the masses of many of these PNGB's, they can present a phenomenological challenge for the model. It will be natural to search further for these at the LHC.

\section{Summary}

The question of the origin of electroweak symmetry is still not answered, and dynamical EWSB via technicolor and extended technicolor remains an interesting possibility. This approach is strongly constrained by flavorchanging neutral current data and precision electroweak measurements. We have reviewed here some recent progress on TC/ETC models. Clearly, there are a number of challenges for such TC/ETC models. Soon, experiments at the LHC will show whether these ideas are realized in nature.

\section{Acknowledgments}

I would like to thank my collaborators on works discussed here, T. Appelquist and M. Piai and, in subsequent papers, N. Christensen and M. Kurachi. I also thank M. Harada, M. Tanabashi, and K. Yamawaki, for organizing this SCGT06 conference. Our research was partially supported by the grant NSF-PHY-03-54776.

\section{References}

1. S. Weinberg, Phys. Rev. D 19, 1277 (1979); L. Susskind, Phys. Rev. D 20, 2619 (1979); see also S. Weinberg, Phys. Rev. D 13, 974 (1976).

2. S. Dimopoulos and L. Susskind, Nucl. Phys. B 155, 237 (1979); E. Eichten and K. Lane, Phys. Lett. B 90, 125 (1980).

3. Some recent reviews are K. Lane, hep-ph/0202255; C. Hill and E. Simmons, Phys. Rep. 381, 235 (2003); R. S. Chivukula, M. Narain, J. Womersley, in http://pdg.lbl.gov

4. T. Appelquist and R. Shrock, Phys. Lett. B548, 204 (2002).

5. T. Appelquist and R. Shrock, Phys. Rev. Lett. 90, 201801 (2003).

6. T. Appelquist, M. Piai, and R. Shrock, Phys. Rev. D 69, 015002 (2004).

7. T. Appelquist, M. Piai, and R. Shrock, Phys. Lett. B 593, 175 (2004); ibid., 595, 442 (2004).

8. T. Appelquist, N. Christensen, M. Piai, and R. Shrock, Phys. Rev. D 70, 093010 (2004).

9. N. Christensen and R. Shrock, Phys. Rev. Lett. 94, 241801 (2005).

10. N. Christensen and R. Shrock, Phys. Rev. D 72, 035013 (2005).

11. N. Christensen and R. Shrock, Phys. Lett. B 632, 92 (2006).

12. N. Christensen and R. Shrock, Phys. Rev. D 74, 015004 (2006). 
13. M. Kurachi and R. Shrock, Phys. Rev. D 74, 056003 (2006).

14. M. Kurachi and R. Shrock, JHEP 12, 034 (2006).

15. M. Kurachi, in these SCGT06 proceedings.

16. P. Sikivie, L. Susskind, M. Voloshin, and V. Zakharov, Nucl. Phys. B 173, 189 (1980)

17. See, e.g., E. Farhi and L. Susskind, Phys. Rept. 74, 277 (1981).

18. B. Holdom, Phys. Lett. B 150, 301 (1985).

19. K. Yamawaki, M. Bando, and K. Matumoto, Phys. Rev. Lett. 56, 1335 (1986).

20. T. Appelquist, D. Karabali, and L. C. R. Wijewardhana, Phys. Rev. Lett. 57, 957 (1986); T. Appelquist and L. C. R. Wijewardhana, Phys. Rev. D 35, 774 (1987); Phys. Rev. D 36, 568 (1987).

21. T. Appelquist and J. Terning, Phys. Rev. D 50, 2116 (1994).

22. T. Appelquist, J. Terning, and L. C. R. Wijewardhana, Phys. Rev. Lett. 77, 1214 (1996); T. Appelquist, A. Ratnaweera, J. Terning, and L. C. R. Wijewardhana, Phys. Rev. D 58, 105017 (1998).

23. V. Miransky and K. Yamawaki, Phys. Rev. D 55, 5051 (1997).

24. R. S. Chivukula, Phys. Rev. D 55, 5238 (1997).

25. M. Peskin and T. Takeuchi, Phys. Rev. Lett. 65, 964 (1990); Phys. Rev. D 46, 381 (1992).

26. K. Lane and A. Martin, Phys. Rev. D 71, 015011 (2005).

27. R. Cahn and M. Suzuki, Phys. Rev. D 44, 3641 (1991); T. Appelquist and G. Triantaphyllou, Phys. Lett. B 278, 345 (1992); R. Sundrum and S. Hsu, Nucl. Phys. B 391, 127 (1993); T. Appelquist and F. Sannino, Phys. Rev. D 59, 067702 (1999); S. Ignjatovic, L. C. R. Wijewardhana, and T. Takeuchi, Phys. Rev. D 61, 056006 (2000).

28. M. Harada, M. Kurachi, and K. Yamawaki, Prog. Theor. Phys. 115, 765 (2006).

29. D. K. Hong and H.-U. Yee, Phys. Rev. D 74, 015011 (2006); J. Hirn and V. Sanz, Phys. Rev. Lett. 97, 121803 (2006); M. Piai, hep-ph/0608241.

30. E. Eichten and K. Lane, Phys. Lett. B 222, 274 (1988).

31. D. Hong, S. Hsu, and F. Sannino, Phys. Lett. B 597, 89 (2004); F. Sannino and K. Tuominen, Phys. Rev. D 71, 051901 (2005).

32. T. Appelquist, N. Evans, and S. Selipsky, Phys. Lett. B 374, 145 (1996).

33. M. Harada, M. Kurachi, and K. Yamawaki, Phys. Rev. D 68, 076001 (2003). 